\title{
Forecasting the investment and production potential of an enterprise of the energy sector
}

\author{
Roman Golov ${ }^{1, *}$, Maria Pushkareva ${ }^{1}$, Larisa Prykina $^{2}$, Oksana Belyantseva ${ }^{3}$, Elena \\ Smorodina $^{3}$ and Denis Sysoev ${ }^{3}$ \\ ${ }^{1}$ Moscow Aviation Institute, Volokolamskoe highway, 4, Moscow, 125993, Russia \\ ${ }^{2}$ Moscow State University of Civil Engineering, 26, Yaroslavskoye sh., Moscow, 129337, Russia \\ ${ }^{3}$ Voronezh State Technical University, Moscow Avenue, 14, Voronezh, 394026, Russia
}

\begin{abstract}
The purpose of this paper is to develop a mechanism for forecasting the potential of an enterprise through the formation of an algorithm for assessing the current state and forecasting investment and production capabilities. The study summarized and systematized theoretical and methodological research in the field of development of management processes, classified the main problems of effective functioning of organizations, developed a method of forecasting the activities of organizations, taking into account the most important criteria. The developed method of the medium-term forecast of the production and financial activities of the organization is universal and can be adapted to the specifics of the activities of any production enterprise.
\end{abstract}

\section{Introduction}

Effective management of an organization implies forecasting its production and financial activities, scope of work and labor productivity, assessing the current state of the business and production capabilities. Forecasting is an active tool for regulating the activities of organizations, allowing one to optimize planned values and build a fourscenario plan for the development of indicators of their activities.

The efficiency of the functioning of industrial enterprises largely depends on the timely resolution of problems. Only after an exact identification of the problem, a research plan can be developed in order to obtain the necessary information to formulate a goal. The draft plan for the study of market conditions, changes in the external environment, the risks of forecast indicators largely depends on how well known the problem is [1].

\section{Materials and methods}

All the main problems of the effective functioning of the enterprise are classified in the paper by the following groups (Fig. 1.): state regulation; organizational and economic nature; social nature; market nature.

\footnotetext{
*Corresponding author: roman_golov@rambler.ru
} 


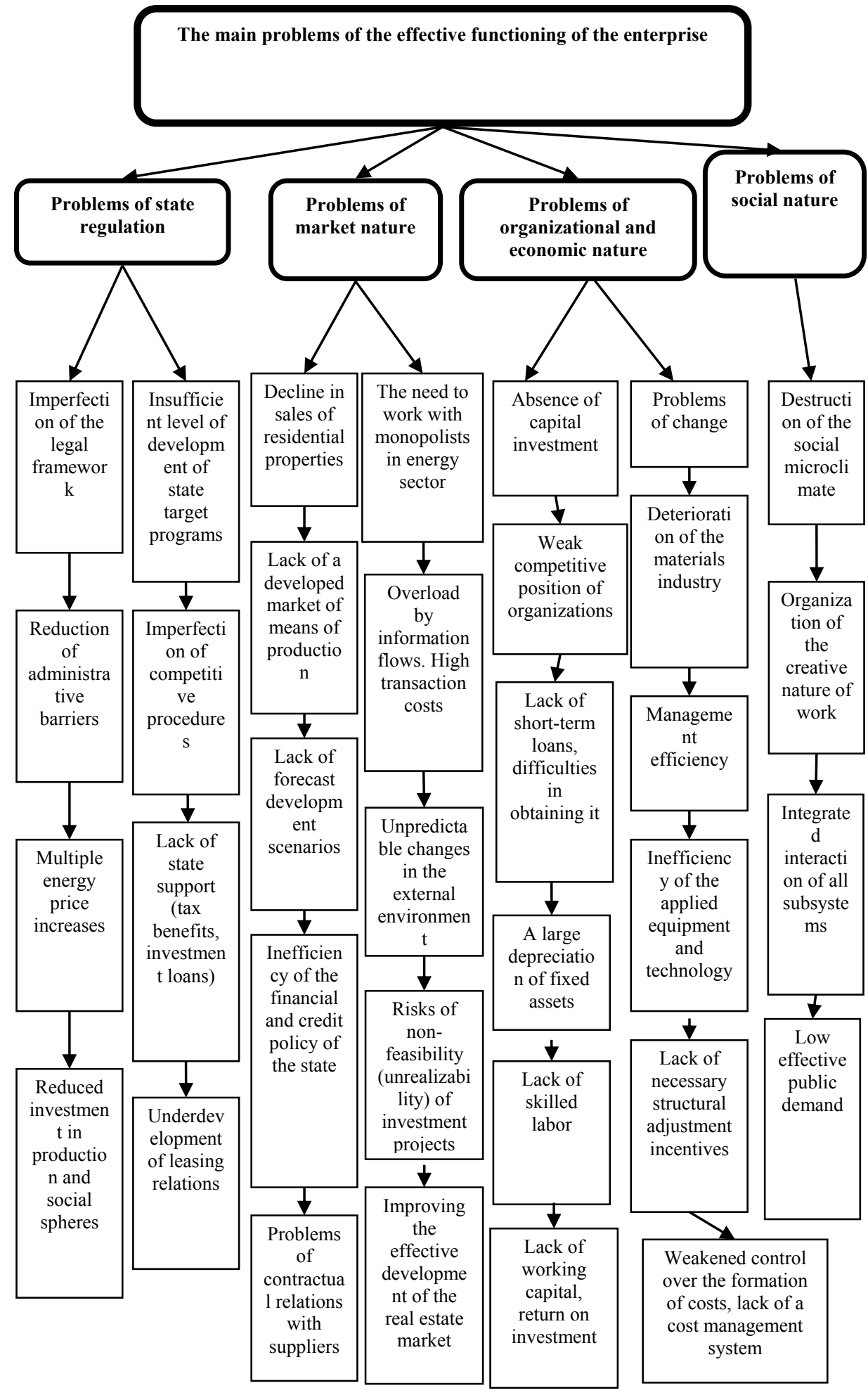

Fig. 1. Classification of problems of effective functioning of enterprises. 
The above classification shows that it is advisable to carry out the forecasting of the market capacity by three main approaches that can be implemented using the appropriate methods of forecast calculations (Fig. 2).

The economic, mathematical, and regulatory approaches based on scientifically based forecasting methods are best suited for forecasting market capacity [2].

From the economic and mathematical methods for assessing the market capacity, we choose the formation of trend models that are based on the mathematical alignment of the dynamic range of the actual values of the total consumption of a particular product for a certain period of time:

$$
E=f(t)
$$

where $\mathrm{E}$ - capacity of the market of specific products; $\mathrm{t}$ - time factor.

The calculated trend models provide the possibility of extrapolating the identified dependencies for the forecasted periods of time in order to obtain predictive estimates of market capacity. The choice of a specific form of functional dependence is carried out on the basis of the calculation of the coefficients reflecting the degree of compliance of the values of market capacity, obtained by the actual values of the trend model. However, in many cases, the actual values of the market capacity in the past period, used to build the trend model, cannot be approximated by any one of the traditional functional dependencies (linear, polynomial, power, etc.). For all products for a sufficiently long time, there are significant changes in the rate of increase or decrease in the market capacity.

To change the sales volume of a specific product group by the stages of market life cycle, functional dependencies are used, approximating cumulative market sales accumulated at each point in time using S-curves.

The essence of the methods used in the paper is that the value of the market capacity is represented as a function of one or several factors. This allows organizations-producers of specific products to identify the quantitative effects of changes in factors on the value of the market capacity of their products, to predict changes in the scale and duration of the stages of the market life cycle, and to effectively respond to changes in market conditions.

The main methods of market capacity forecasting, recommended by us for its use in the practice of the organization:

1. Methods for studying consumer opinions and collective expert assessment (Delphi method, independent composite forecast). Forecasts are based on identifying the opinions of people involved in the process of buying and selling (consumers, employees of marketing departments, salespeople, managers and specialists of research, design, technology and production departments of the organization).

2. Methods for analyzing time series should be applied in the presence of statistical data on sales volumes for previous periods. Using them, the volume of sales is presented as a function of time, not taking into account other factors affecting demand. In this regard, the estimates obtained using these methods must be adjusted to the opinion of experts.

3. Correlation and regression methods should be used more correctly for long-term forecasts of the development of an organization, which is explained by the influence of seasonal factors on the accuracy of short-term forecasts. Correlation and regression methods are used in identifying one or more factors affecting the value of market demand. In this case, the statistical dependence of the market capacity on these factors is calculated. 


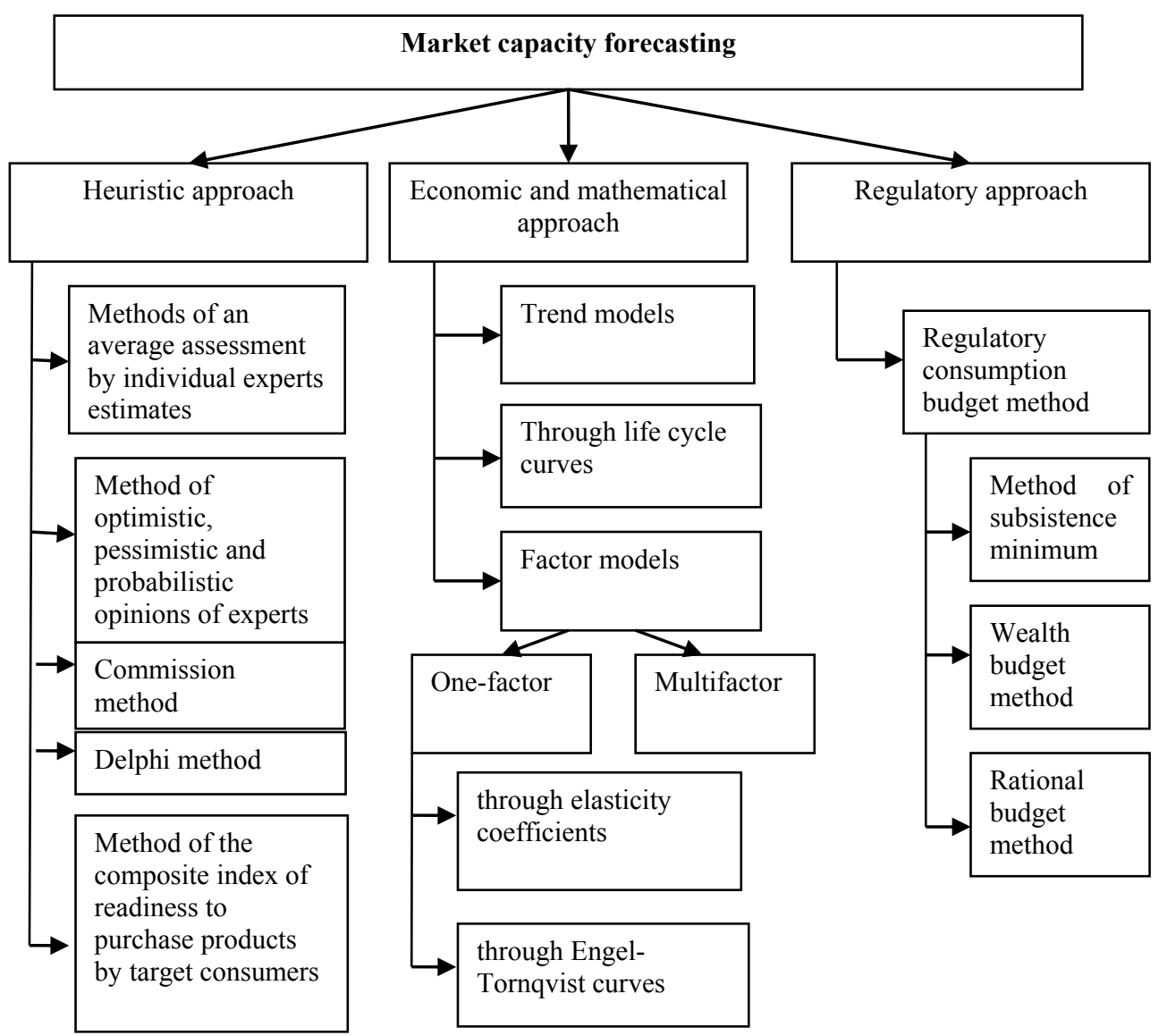

Fig. 2. Approaches and methods of forecasting the capacity of product market.

\section{Results}

The study of market capacity or market demand implies the determination of sales volume in the selected market of certain products for a specific period of time. Our studies show that data on the market capacity and the share of individual manufacturers are necessary both for expanding the position of an organization already holding a stable position in the market and for penetrating of a new organization to the market.

The application of the predictive model taking into account the requirements of adaptability (flexibility, reliability of development, elasticity) allows organizations to adapt to changing operating conditions, changing not only quantitative characteristics but also quality $[3,4]$.

The adaptive methods are the methods that are able to a greater extent take into account the current information in the market at the present time, and to a lesser extent - the past information. The value of such methods, in our opinion, lies in the free change of the constructed model when new information comes from the environment, moreover, if they do not require the division of the dynamic series into components and separate prediction of individual components.

The developed method of forecasting and distributing the financial results of an organization includes the following steps: 
- calculation of profit and profitability projection;

- verification of the profitability of the profit plan and calculating the marginal rate of its consumption;

- implementation of the results of the assessment and forecasting of the financial results of the organization.

The method is based on the mechanism of formation and distribution of financial results, which is an element of the organization's management system (Fig. 3).

Economic mechanism of the production enterprise

Financial mechanism of the production enterprise

The mechanism of formation and distribution of financial results:

- institutional link - a group of financial workers engaged in

accounting for financial results;

- functional element - principles, forms, methods, technologies,

incentives, fines;

- legal unit - a system of legislative acts regulating the process of

formation and distribution of financial results.

Fig. 3. The mechanism of formation and distribution of financial results.

The developed method of forecasting the scope of work and labor productivity of the organization is based on the use of approximating functions and linear, parabolic, power, and exponential dependencies $[5,6]$.

The tendency of development of enterprise performance indicators will not be pronounced, and in this case, the function should be selected by linear, parabolic, power, and exponential regression equations with subsequent comparison of calculated workload and performance values $\left(\mathrm{R}^{2}\right)$. All the specified functions are included in the search. The final choice of the trend equation is based on the rationale for the connection forms chosen for building our model. The calculations are summarized in Table 1.

Many years of experience of many researchers shows that the linear trend form gives a correct idea of the evolution of indicators. No matter how complicated the tendency of the series and no matter how bad the approximation given by a line, the best in the sense of the method of least squares, a linear equation should be applied to express the average annual growth (average speed) [7, 8].

Table 1. Parameterized trend conditions and the values of the coefficient of determination for the considered interdependencies.

\begin{tabular}{|c|c|c|c|c|}
\hline \multirow{2}{*}{$\begin{array}{c}\text { Type of } \\
\text { dependence }\end{array}$} & \multicolumn{2}{|c|}{ Parameterized trend conditions } & \multicolumn{2}{c|}{ Coefficient of determination } \\
\cline { 2 - 5 } & Scope of contract work & Labor productivity & $\begin{array}{c}\text { Scope of } \\
\text { contract work }\end{array}$ & $\begin{array}{c}\text { Labor } \\
\text { productivity }\end{array}$ \\
\hline Linear & $\mathrm{y}=4077.3 \mathrm{x}-8146982$ & $\mathrm{y}=31.506 \mathrm{x}-62751$ & 0.57 & 0.17 \\
\hline Parabolic & $\begin{array}{c}\mathrm{y}=-1154.73 \mathrm{x}^{2}+ \\
4644935.04 \mathrm{x}-\end{array}$ & $\begin{array}{c}\mathrm{y}=-40.78464236 \mathrm{x}^{2}+ \\
163944.9854 \mathrm{x}- \\
164754701.17\end{array}$ & 0.67 & 0.79 \\
\hline
\end{tabular}




\begin{tabular}{|c|c|c|c|c|}
\hline Power & $\mathrm{y}=\mathrm{e}^{-1512.46} \mathrm{x}^{200.27}$ & $\mathrm{y}=\mathrm{e}^{-143.54} \mathrm{x}^{0.0746}$ & 0.55 & 0.23 \\
\hline Exponential & $\mathrm{y}=4.97 * 10^{-83} \mathrm{e}^{0.0996 \mathrm{x}}$ & $\mathrm{y}=5 \mathrm{E}-63 \mathrm{e}^{0.0746 \mathrm{x}}$ & 0.55 & 0.23 \\
\hline
\end{tabular}

Note: $y$ - predicted value; $x$ - number of the year.

The method of forecasting the organization's production capabilities involves assessing the actual capacity, assessing the effective capacity, and assessing the design capacity of the company (Table 2). The main stages of assessing the organization's production capabilities are presented in Fig. 4.

Table 2. The main components of the methodology for forecasting the production capabilities of the organization.

\begin{tabular}{|c|c|}
\hline Main components & \multicolumn{1}{c|}{ Content } \\
\hline $\begin{array}{c}\text { Assessment of the actual } \\
\text { capacity }\end{array}$ & $\begin{array}{l}\text { Operative planning. Preventive equipment repair. Operative and } \\
\text { medium-term planning. Elimination of "bottlenecks" in equipment } \\
\text { and personnel by various organizational measures. }\end{array}$ \\
\hline $\begin{array}{c}\text { Assessment of the } \\
\text { effective capacity }\end{array}$ & $\begin{array}{l}\text { Operative and medium-term planning. Elimination of "bottlenecks" } \\
\text { in equipment and personnel by organizational and investment } \\
\text { measures: changing the form of specialization, drawing up and } \\
\text { observing the work schedule, carrying out repairs, equipment, } \\
\text { flexible work schedule, and outsourcing. }\end{array}$ \\
\hline $\begin{array}{c}\text { Assessment of the design } \\
\text { capacity }\end{array}$ & $\begin{array}{l}\text { Medium and long term planning. Elimination of "bottlenecks" by } \\
\text { attracting investments in replacement and modernization of } \\
\text { equipment. }\end{array}$ \\
\hline
\end{tabular}

The calculation of production program variants is supposed to be carried out using linear programming, where the upper limits - the total time base, the lower limits - the nonnegative values of the time norms, the objective function - the maximization of the operating time of the production equipment.

Task setting is presented in a formalized form:

$$
\begin{gathered}
F(X)=\sum_{j=1}^{m} \sum_{i=1}^{n} a_{j . i} X_{i} \Rightarrow \max , \quad \text { when } \\
a_{1.1} x_{1}+a_{1.2} x_{2}+\ldots+a_{1 . n} x_{n} \leq b_{1} \\
a_{2.1} x_{1}+a_{2.2} x_{2}+\ldots+a_{2 . n} x_{n} \leq b_{2} \\
a_{m .1} x_{1}+a_{m .2} x_{2}+\ldots+a_{m . n} x_{n} \leq b_{m}
\end{gathered}
$$

where:

$x_{i}$ - the number of works of a certain type;

$a_{j . i}$ - rate of time for implementation of work;

$b_{j}$ - the general time limit of equipment operation.

An additional condition to be taken into account is the integrality of the solution, i.e. the number of works is a multiple of one.

Forecasting the production capabilities of the organization under design capacity conditions involves identifying and eliminating the "bottlenecks" in actual and effective capacity. Market restriction - restriction on the volume of demand for the products of organizations. To eliminate "bottlenecks", it is necessary to determine the minimum deviations between the time limit and the optimal loading time. 


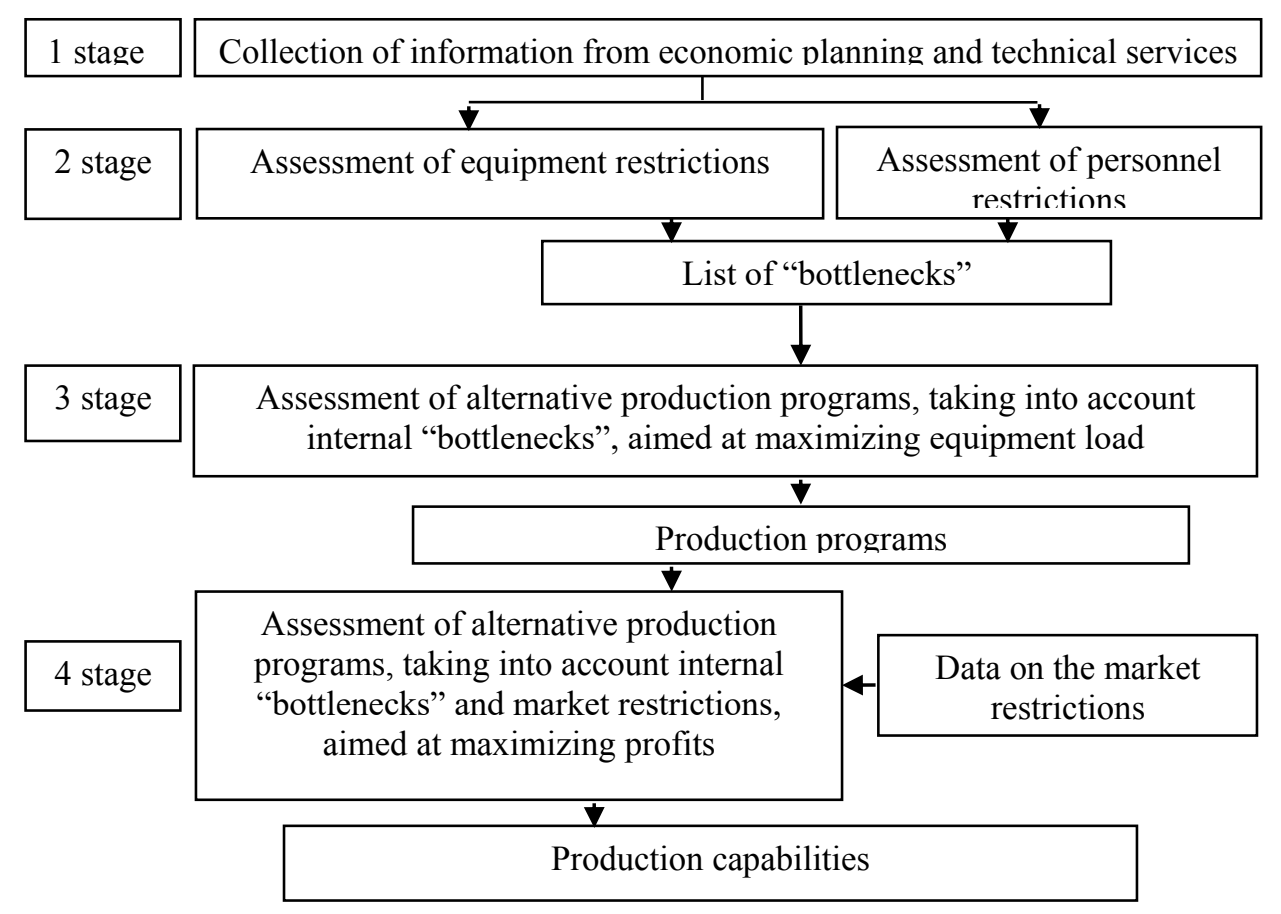

Fig. 4. The main stages of forecasting the production capabilities of the organization.

\section{Conclusion}

The proposed method of forecasting the investment and production potential of an enterprise allows identifying design, effective, and actual capacities in physical and value indicators, as well as finding "bottlenecks" and calculating the costs of their elimination. The method contributes to the search for optimal loading options for fixed assets and equipment of the organization.

\section{References}

1. C. Furlong, S. De Silva, K. Gan, L. Guthrie, R. Considine, Journal of Environmental Management 191, 83-95 (2017)

2. K. Anagnostis, K. Alexios, Procedia Economics and Finance 9, 120-132 (2014)

3. L. Shan, A. Yu, Y.Wu, Habitat International 59, 90-100 (2017)

4. S. Gupta, N. K. Malhotra, Journal of Business Research 69, 5671-5681 (2016)

5. T.W. Chung, The Asian Journal of Shipping and Logistics 32, 257-264 (2016)

6. N.G. Bagautdinova, A.V. Sarkind, I.R. Gafurovc, Procedia Economics and Finance 14, 23-29 (2014)

7. P. Ovchinnikov, A. Borodinecs, K. Strelets, Building and Environment 112, (2017) doi:10.1016/j.buildenv.2016.11.029

8. J. Enqvist, M. Graham, J. Nikkinen. Research in International Business and Finance 32, 36-49 (2014) 